\title{
Estudo da conservação de tomates "sweet grape" com revestimentos formulados a partir de fécula de mandioca e subprodutos da vinificação
}

\author{
Elizangela Gonçalves de Oliveira, Tatiana Valesca Rodriguez Alicieo
}

https://doi.org/10.4322/mp.978-65-991393-5-2.c9

\section{Resumo}

O presente trabalho teve por objetivo avaliar o efeito de coberturas filmogênicas de fécula de mandioca e bagaço de uva na conservação de tomate "sweet grape", armazenados à temperatura ambiente e em refrigeração. Foram elaboradas duas soluções filmogênicas de gelatina, uma a base de fécula de mandioca e outra a base de bagaço de uva, ambas com concentração de 3\%, as quais foram misturadas a uma solução de gelatina. As duas soluções filmogênicas foram testadas em amostras de tomates previamente selecionados, lavados e sanitizados. As amostras de tomate foram divididas em grupos distintos: grupo de controle, grupo revestido com biofilme de fécula de mandioca e grupo revestido com biofilme de bagaço de uva, e subdivididas entre amostras mantidas em temperatura ambiente e amostras mantidas sob refrigeração. Após doze dias de avaliação, os efeitos dos tratamentos foram verificados por meio da perda de massa (\%), análise colorimétrica e análise microbiológica. Foi constatada a eficácia de ambas as variedades de biofilme para reduzir a perda de massa nas amostras mantidas em temperatura ambiente, com resultados sem diferença significativa pelo teste de Tukey entre as duas coberturas. Contudo, as amostras revestidas com biofilme de fécula de mandioca, sob temperatura ambiente, apresentaram resultados significativamente inferiores na contagem microbiana sob aspectos quantitativos, evidenciando eficácia qualitativamente inferior do biofilme de bagaço de uva. No entanto, ambas as coberturas se mostraram prejudiciais a proteção contra perda de massa sob condições de refrigeração, apresentando taxas de perdas superiores as das amostras controle.

Palavras-chave: cobertura filmogênica, polímeros, refrigeração, revestimento.

\section{Introdução}

Devido à preocupação com a questão da redução dos impactos ambientais, muitas pesquisas vêm sendo realizadas a fim de aperfeiçoar a produção de materiais biodegradáveis, a partir de fontes renováveis. Um exemplo disso é a produção de filmes biodegradáveis ou biofilmes, que se apresentam como uma alternativa para a redução dos impactos provocados pelo uso de polímeros derivados do petróleo, devido ao fato de poderem ser produzidos a partir de materiais renováveis como proteínas e polissacarídeos (PETRIKOSKI, 2013). 
O desenvolvimento de filmes comestíveis e coberturas com permeabilidade seletiva pode ser extremamente promissor no controle de mudanças respiratórias e aumento do período de conservação de produtos vegetais frescos (SILVA et al., 2012). O uso de coberturas comestíveis na conservação de frutas e hortaliças na condição póscolheita tem sido preconizado como uma tecnologia emergente e de grande potencial.

A fécula é um dos produtos mais importantes obtidos a partir da mandioca. Apesar do amido e da fécula possuírem a mesma estrutura química, pode-se distinguir os dois devido ao fato de a fécula ser obtida de matérias-primas subterrâneas, enquanto o amido tem uma definição mais geral, pois pode ser obtido de qualquer parte da planta (CEREDA, 2005). Vários estudos realizados avaliam a utilização dos filmes de fécula de mandioca obtidos a partir de uma suspensão que possui apenas o polímero e o solvente, sendo este mais comumente a água. No entanto, na tentativa de aperfeiçoar as propriedades dos biofilmes podem ser elaborados filmes compostos adicionando à solução plastificantes e aditivos que contribuam positivamente nas propriedades mecânicas e de barreira ou ainda utilizando uma combinação de biopolímeros agregando as características positivas de cada um dos componentes utilizados (FAKHOURI et al., 2007; ROJAS-GRAÜ; SOLIVA-FORTUNY; MARTÍN-BELLOSO, 2009).

O biofilme é produzido a partir de materiais biológicos, agindo como barreira a elementos externos e protegendo o produto embalado de danos físicos e biológicos, aumentando assim sua vida útil. O desenvolvimento desses biofilmes tem avançado consideravelmente, devido ao interesse por alimentos de alta qualidade, à preocupação ambiental e às oportunidades em criar mercados para as matériasprimas formadoras de filme, derivadas de produtos agrícolas (REIS et al., 2011).

O bagaço é o principal resíduo obtido do processo de vinificação, sendo também o mais volumoso. É definido como o produto resultante da prensagem das uvas frescas, fermentadas ou não, sendo composto pelas cascas da uva, sementes e engaço. $O$ conjunto mosto/vinho que embebe as partes sólidas da uva também pode ser componente do bagaço de uva (OLIVEIRA, 2010; RUGGIERI et al., 2009). As cascas representam em média $82 \%$ do bagaço de uva (peso úmido) após a etapa de prensagem do processo de vinificação (JIANG; SIMONSEN; ZHAO, 2011). Estudos avaliaram a composição centesimal das mesmas e obtiveram valores próximos a 55\% de fibras, $12 \%$ de proteínas, $6 \%$ de lipídios, $7 \%$ de cinzas e $2 \%$ de açúcares solúveis (DENG; PENNER; ZHAO, 2011; BAUMGÄRTEL et al., 2007). Da quantidade de fibras presente na casca, quase 95\% são consideradas insolúveis, sendo que as principais fibras encontradas são pectina, celulose e lignina. A proporção destas pode variar muito e depende do cultivar estudado, entre outros fatores.

Deng, Penner e Zhao (2011) desenvolveram filmes biodegradáveis a base de extratos de bagaço de uvas provenientes do processo de vinificação. Através do desenvolvimento de diferentes formulações de filmes, que além dos extratos de uva continham pectina, polissacarídeos, alginato e/ou uma mistura comercial de polissacarídeos, o estudo demonstra a possibilidade de obter filmes com atividade antimicrobiana, antioxidante e com boas propriedades mecânicas a partir de resíduos vitivinícolas.

O tomate apresenta elevado conteúdo de água, estando sujeito às variações de temperatura e umidade relativa do ambiente onde se encontra. A perda de água ocasiona perda de massa e altera a aparência do fruto (MARCOS, 2001). A utilização 
de películas comestíveis para revestir produtos hortifrutícolas mostra-se eficaz na redução de perda de água e manutenção da qualidade do fruto.

Segundo Luvielmo e Lamas (2013) o uso de revestimentos comestíveis, aplicado em frutas, é uma tecnologia economicamente interessante, uma vez que são utilizadas pequenas quantidades de matérias-primas e muitas destas de baixo valor comercial, como a fécula de mandioca. Apesar de existir uma grande variedade de revestimentos comestíveis e muitos estudos com aplicações em frutas, ainda é um campo a ser explorado.

O objetivo do presente estudo foi avaliar o efeito de coberturas filmogênicas de gelatina incorporadas de fécula de mandioca e bagaço de uva na conservação tomate "sweet grape", armazenados a temperatura ambiente e em refrigeração.

\section{Materiais e Métodos}

Foram avaliados frutos de tomates "sweet grape" maduros adquiridos em mercado varejista de produtos alimentícios. Os frutos foram selecionados em vista do tamanho, coloração (vermelha), firmeza ao tato e ausência de injúrias, sendo lavados em água corrente e sanitizados com solução de hipoclorito de sódio a $200 \mathrm{mg} \mathrm{L}^{-1}$, por $15 \mathrm{~min}$. Após esse período foram retirados da solução e colocados em peneiras para a secagem completa em temperatura ambiente.

A fécula de mandioca utilizada nos experimentos foi obtida em mercado varejista de alimentos. Já o bagaço de uva (Vitis vinífera, variedade Merlot, safra 2017-2018), utilizado para o desenvolvimento dos biofilmes foi doado pela Vinícola Peruzzo localizada na cidade de Bagé/RS. O bagaço de uva foi congelado a $-18{ }^{\circ} \mathrm{C}$ e para a realização dos ensaios as amostras foram descongeladas em refrigeração a $7^{\circ} \mathrm{C}$ e as sementes foram separadas das cascas manualmente. As cascas foram secas em estufa na temperatura de $70{ }^{\circ} \mathrm{C}$ por aproximadamente $2 \mathrm{~h}$ segundo metodologia proposta por Deamici et al. (2016). Após a secagem, as amostras foram moídas em moinho analítico e acondicionadas em temperatura ambiente até a realização dos experimentos.

Após a sanitização e secagem, os frutos foram divididos em três grupos: amostras controle, sem aplicação de biofilme; amostras submetidas a aplicação de biofilme de fécula de mandioca com concentração de $3 \%$, misturada com solução de gelatina incolor a 10\% de concentração; e amostras recobertas com biofilme de bagaço de uva feito sob as mesmas condições de concentração do outro biofilme. A concentração de $3 \%$ foi definida a partir dos resultados obtidos por Lemos et al. (2008), que avaliaram a perda de massa de soluções filmogênicas de fécula de mandioca aplicada a pimentões e obteve melhores taxas de redução de perdas nesta concentração em amostras não refrigeradas.

Para a aplicação dos revestimentos, os frutos foram imersos por 1 min e, em seguida, suspensos e deixados secar naturalmente, sendo posteriormente acomodados em bandejas de poliestireno. Metade das amostras de cada um dos grupos foi armazenada em temperatura ambiente a $20 \pm 1{ }^{\circ} \mathrm{C}$ e $68 \pm 2 \%$ UR. A outra metade foi, por sua vez, colocada sob refrigeração controlada a $4 \pm 1{ }^{\circ} \mathrm{C}$ e $68 \pm 2 \%$ UR. Houve 0 acompanhamento da perda de massa e da cor das amostras durante 12 dias. A perda de massa dos frutos foi avaliada em todos os períodos de armazenamento, com 
auxílio de balança analítica com precisão de 0,0001 g e os resultados expressos em porcentagem em relação à massa inicial conforme a equação 1.

$\%$ Perda de massa $\frac{\left(m_{0}-m_{i}\right)}{m_{0}} \times 100 \%$

(Equação 1)

Sendo: $\mathrm{m}_{0}$ a massa inicial $(\mathrm{g})$ e $\mathrm{m}_{\mathrm{i}}$ a massa a cada intervalo de tempo $(\mathrm{g})$

A análise de cor foi realizada com um colorímetro Minolta a partir de um diagrama tridimensional de cores $\left(L^{*}, a^{*}, b^{\star}\right)$, em que $L$ indica luminosidade, $a^{*}$ indica cromaticidade tendendo do verde (-) até vermelho $(+)$ e $b^{*}$ indica a cromaticidade que varia do azul (-) até amarelo (+). As análises foram realizadas em triplicata, para maior confiabilidade dos resultados. As diferenças significativas entre as médias $(p<0,05)$ foram analisadas pelo teste de Tukey, utilizando-se o programa Statistica 6.0 (StatSoft Inc., Tulsa, Okla, USA).

As determinações microbiológicas foram realizadas de acordo com as recomendações de Downes e Ito (2001). A enumeração de bactérias mesófilas aeróbias foi realizada utilizando-se semeadura em ágar para contagem total (Plate Count Agar - PCA , MERCK ${ }^{\circledR}$ ) com incubação a $37^{\circ} \mathrm{C}$ por $24 \mathrm{~h}$.

\section{Resultados e Discussão}

$\mathrm{Na}$ Tabela 1 são apresentados os resultados de perda massa (\%) para as amostras recobertas com as coberturas de fécula de mandioca e bagaço de uva nas condições de temperatura ambiente e refrigerada.

Tabela 1. Valores médios para perda de massa (\%) de frutos de tomate "sweet grape" revestidos com biofilme de fécula de mandioca e bagaço de uva armazenados sob refrigeração.

\begin{tabular}{|c|c|c|c|c|c|c|c|}
\hline \multicolumn{8}{|c|}{ Temperatura ambiente ${ }^{\star}$} \\
\hline Tratamento/Dias ** & $\mathbf{0}$ & 1 & 3 & 5 & 8 & 10 & 12 \\
\hline Controle (T1) & 0 & $\begin{array}{l}0,408 \mathrm{a} \\
\pm 0,093\end{array}$ & $\begin{array}{l}3,938 a \\
\pm 0,672\end{array}$ & $\begin{array}{l}6,568 \mathrm{a} \\
\pm 2,069\end{array}$ & $\begin{array}{l}7,093 a \\
\pm 2,409\end{array}$ & $\begin{array}{l}8,142 a \\
\pm 2,101\end{array}$ & $\begin{array}{l}9,157 a \\
\pm 1,865\end{array}$ \\
\hline Féc. Mandioca (T2) & 0 & $\begin{array}{l}1,274 b \\
\pm 0,152\end{array}$ & $\begin{array}{l}2,620 b \\
\pm 0,353\end{array}$ & $\begin{array}{l}4,850 \mathrm{~b} \\
\pm 0,787\end{array}$ & $\begin{array}{l}6,237 \mathrm{~b} \\
\pm 1,061\end{array}$ & $\begin{array}{l}6,141 \mathrm{~b} \\
\pm 1,211\end{array}$ & $\begin{array}{l}7,050 \mathrm{~b} \\
\pm 2,409\end{array}$ \\
\hline Bagaço de uva (T3) & 0 & $\begin{array}{l}1,235 b \\
\pm 0,122\end{array}$ & $\begin{array}{r}3,627 b \\
\pm 0,893\end{array}$ & $\begin{array}{l}3,410 \mathrm{~b} \\
\pm 0,351\end{array}$ & $\begin{array}{l}5,366 \mathrm{~b} \\
\pm 0,410\end{array}$ & $\begin{array}{l}6,240 \mathrm{~b} \\
\pm 0,453\end{array}$ & $\begin{array}{l}7,140 \mathrm{~b} \\
\pm 0,333\end{array}$ \\
\hline \multicolumn{8}{|c|}{ Refrigeração* } \\
\hline Tratamento/Dias ** & 0 & 1 & 3 & 5 & 8 & 10 & 12 \\
\hline Controle (T4) & 0 & $\begin{array}{l}0,355 a \\
\pm 0,076\end{array}$ & $\begin{array}{l}0,659 a \\
\pm 0,262\end{array}$ & $\begin{array}{l}0,779 a \\
\pm 0,242\end{array}$ & $\begin{array}{l}1,044 a \\
\pm 0,271\end{array}$ & $\begin{array}{l}2,012 a \\
\pm 0,439\end{array}$ & $\begin{array}{l}2,299 \\
\pm 0,484\end{array}$ \\
\hline Féc. Mandioca (T5) & 0 & $\begin{array}{l}0,800 b \\
\pm 0,315\end{array}$ & $\begin{array}{l}1,466 b \\
\pm 0,355\end{array}$ & $\begin{array}{l}2,453 b \\
\pm 0,413\end{array}$ & $\begin{array}{l}2,903 b \\
\pm 0,428\end{array}$ & $\begin{array}{l}3,533 b \\
\pm 0,485\end{array}$ & $\begin{array}{l}4,055 b \\
\pm 0,267\end{array}$ \\
\hline Bagaço de uva (T6) & 0 & $\begin{array}{l}0,210 a \\
\pm 0,122\end{array}$ & $\begin{array}{l}1,157 b \\
\pm 0,103\end{array}$ & $\begin{array}{r}1,694 c \\
\pm 0,080\end{array}$ & $\begin{array}{l}2,879 b \\
\pm 0,135\end{array}$ & $\begin{array}{l}3,197 \mathrm{~b} \\
\pm 0,160\end{array}$ & $\begin{array}{l}3,543 c \\
\pm 0,126\end{array}$ \\
\hline
\end{tabular}

*média \pm desvio padrão. ${ }^{* * L e t r a s ~ m i n u ́ s c u l a s ~ d i f e r e n t e s ~ n a ~ m e s m a ~ c o l u n a ~ a p r e s e n t a m ~}$ diferença significativa $(p<0,05)$ pelo teste de Tukey. 
A perda de água de produtos armazenados não só resulta em perda de massa, mas também de qualidade. Segundo Chitarra e Chitarra (2005), alguma perda de água pode ser tolerada, mas aquelas responsáveis pelo enrugamento devem ser evitadas. Perdas da ordem de $3 \%$ a $6 \%$ são suficientes para causar um marcante declínio de qualidade, entretanto, alguns produtos são ainda comercializáveis com 10\% de perda de umidade. Observa-se na Tabela 1 que as amostras revestidas com biofilme apresentaram a partir do terceiro dia taxas de perda de água significativamente inferiores às amostras controle. Ao término do experimento, no décimo segundo dia, ambas apresentavam perda de massa de $7 \%$, com perdas $23 \%$ inferiores em relação a amostra controle. Tais resultados foram superiores em relação ao estudo de Lemos et al. (2008), que obteve êxito na redução das taxas de perda apenas no vigésimo dia de avaliação, quando as amostras já registravam perdas de $24 \%$ e já haviam ultrapassado limites de tolerância de 10\% estabelecidos por Chitarra e Chitarra (2005).

Em relação a perda de massa em amostras refrigeradas, a aplicação dos biofilmes foi prejudicial à conservação destas. A amostra de biofilme de bagaço de uva e fécula de mandioca apresentaram perdas superiores em relação a amostra controle refrigerada, respectivamente na proporção de 55\% e 77\%. O mesmo fenômeno, ainda que em menor grau, ocorreu também nos experimentos de Lemos et al. (2008).

Sob aspectos de análise microbiológica, os resultados da Tabela 2 evidenciam excelente desempenho do biofilme de fécula de mandioca, mantendo níveis mínimos de contaminação nas amostras refrigeradas e não refrigeradas. $O$ mesmo não se repetiu nas amostras revestidas por biofilme de bagaço de uva, em que houve mera redução dos níveis de contaminação em relação as amostras controle.

Tabela 2. Análise microbiológica de bactérias mesófilas aeróbias nos frutos de tomate "sweet grape" revestidos com biofilme de fécula de mandioca e bagaço de uva armazenados em condições ambientes e sob refrigeração.

\begin{tabular}{cc}
\hline Tratamento & Contagem (UFC/g) \\
\hline 1 & $3,3 \times 10^{5}$ \\
2 & $<1,0$ \\
3 & $8,75 \times 10^{3}$ \\
4 & $2,14 \times 10^{5}$ \\
5 & $<1,0$ \\
6 & $3,8 \times 10^{3}$ \\
\hline
\end{tabular}

*T1: Condições ambiente - amostra controle; T2: Condições ambiente - fécula de mandioca; T3: Condições ambiente - bagaço de uva; T4: Refrigeração - amostra controle; T5: Refrigeração - fécula de mandioca; T6: Refrigeração - bagaço de uva.

A Tabela 3 mostra as variações do parâmetro $L$, que varia de 0 para preto a 100 para branco. Para todos os tratamentos houve decréscimo no valor de $L$, o escurecimento do fruto provocado pelo processo de maturação. No entanto, observa-se que a cobertura de bagaço de uva provocou menores reduções em seus valores quando comparados com o grupo controle. 
A cobertura de bagaço de uva reteve luminosidade e garantiu maior brilho, quando comparada aos outros tratamentos e a amostra controle. O brilho produzido aos tomates por esta cobertura pode ser observado na Figura 1.

Tabela 3. Valores médios para a cor de frutos de tomate "sweet grape" revestidos com biofilme de fécula de mandioca e bagaço de uva armazenados em condições ambientes e sob refrigeração.

\begin{tabular}{ccccc}
\hline Cor & Tratamento & 0 (dias) & 5 (dias) & 12 (dias) \\
\hline $\mathrm{L}^{*}$ & T1 & $33,02 \pm 1,48 \mathrm{a}$ & $23,20 \pm 1,82 \mathrm{a}$ & $20,39 \pm 2,61 \mathrm{a}$ \\
& T2 & $36,41 \pm 2,36 \mathrm{~b}$ & $35,54 \pm 0,74 \mathrm{~d}$ & $25,74 \pm 1,97 \mathrm{~b}$ \\
& T3 & $33,79 \pm 1,47 \mathrm{a}$ & $33,11 \pm 084 \mathrm{~b}$ & $32,57 \pm 1,25 \mathrm{c}$ \\
& T4 & $33,36 \pm 2,41 \mathrm{a}$ & $30,48 \pm 1,24 \mathrm{c}$ & $29,80 \pm 1,46 \mathrm{c}$ \\
& T5 & $33,52 \pm 1,41 \mathrm{a}$ & $31,44 \pm 1,48 \mathrm{c}$ & $28,86 \pm 2,51 \mathrm{c}$ \\
$\mathrm{a}$ & T6 & $33,78 \pm 1,47 \mathrm{a}$ & $33,11 \pm 0,84 \mathrm{~b}$ & $31,56 \pm 1,25 \mathrm{c}$ \\
& T1 & $15,08 \pm 075 \mathrm{a}$ & $10,63 \pm 2,88 \mathrm{a}$ & $16,38 \pm 1,05 \mathrm{a}$ \\
& T2 & $16,39 \pm 1,44 \mathrm{a}$ & $18,64 \pm 1,25 \mathrm{~b}$ & $13,02 \pm 2,01 \mathrm{~b}$ \\
& T3 & $13,39 \pm 1,53 \mathrm{~b}$ & $15,95 \pm 0,46 \mathrm{c}$ & $16,41 \pm 0,53 \mathrm{a}$ \\
& T4 & $15,83 \pm 081 \mathrm{a}$ & $18,75 \pm 1,41 \mathrm{~b}$ & $15,95 \pm 1,29 \mathrm{a}$ \\
& T5 & $16,76 \pm 1,57 \mathrm{a}$ & $15,00 \pm 2,38 \mathrm{c}$ & $16,11 \pm 1,10 \mathrm{a}$ \\
& T6 & $13,39 \pm 1,53 \mathrm{~b}$ & $15,95 \pm 0,46 \mathrm{c}$ & $16,41 \pm 0,53 \mathrm{a}$ \\
\hline $\mathrm{b}$ * & T1 & $12,20 \pm 0,63 \mathrm{a}$ & $8,24 \pm 1,47 \mathrm{a}$ & $12,84 \pm 1,08 \mathrm{a}$ \\
& T2 & $11,90 \pm 0,89 \mathrm{~b}$ & $14,76 \pm 0,93 \mathrm{c}$ & $11,88 \pm 0,86 \mathrm{a}$ \\
& T3 & $10,11 \pm 1,00 \mathrm{~b}$ & $12,68 \pm 1,11 \mathrm{~b}$ & $12,51 \pm 0,31 \mathrm{a}$ \\
& T4 & $12,77 \pm 0,66 \mathrm{a}$ & $15,37 \pm 0,62 \mathrm{c}$ & $12,32 \pm 0,63 \mathrm{a}$ \\
& T5 & $13,82 \pm 1,43 \mathrm{a}$ & $13,82 \pm 1,43 \mathrm{~b}$ & $13,19 \pm 1,33 \mathrm{~b}$ \\
& T6 & $10,11 \pm 0,99 \mathrm{~b}$ & $12,67 \pm 1,10 \mathrm{~b}$ & $12,51 \pm 0,30 \mathrm{a}$ \\
\hline
\end{tabular}

*T1: Condições ambiente - amostra controle; T2: Condições ambiente - fécula de mandioca; T3: Condições ambiente - bagaço de uva; T4: Refrigeração - amostra controle; T5: Refrigeração - fécula de mandioca; T6: Refrigeração - bagaço de uva. **média \pm desvio padrão. ${ }^{* *}$ Letras minúsculas diferentes na mesma coluna apresentam diferença significativa ( $p$ $<0,05)$ pelo teste de Tukey.

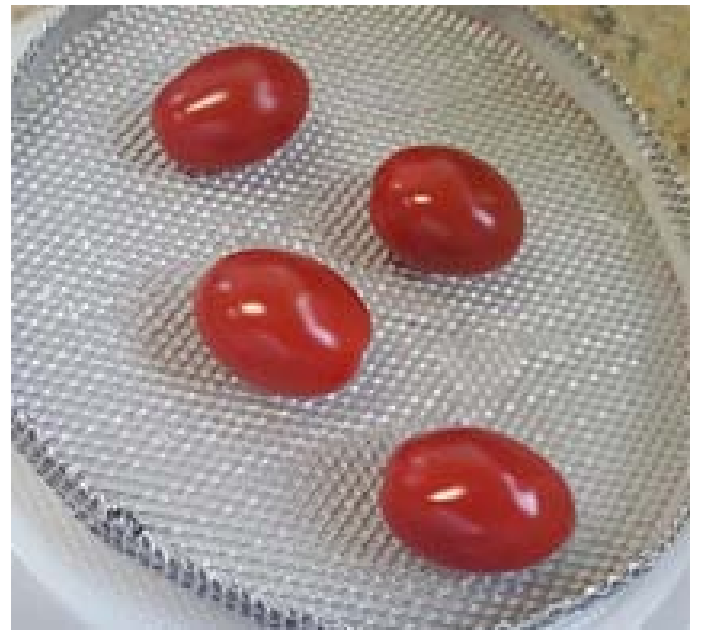

Figura 1. Amostra de tomate "sweet grape" recoberta com solução filmogênica de bagaço de uva $3 \%$. 
Oliveira, Martins e Santos (2012) também observaram reduções na luminosidade (L) de tomates revestidos com pectina em concentrações de $2 \%, 5 \%$ e $8 \%$. Não houve outros efeitos significativos nos demais parâmetros avaliados na Tabela 3.

\section{Conclusão}

O uso de biofilmes de fécula de mandioca e bagaço de uva em duas condições de armazenamento foi avaliado para o aumento da vida útil de tomates "sweet grape".

Tanto o biofilme de fécula de mandioca quanto o de bagaço de uva, na concentração de $3 \%$, foram eficientes em retardar o metabolismo pós-colheita e prolongar a conservação das amostras armazenados em temperatura ambiente, reduzindo taxas de perda de massa. Houve, contudo, vantagem em prol do biofilme de fécula de mandioca em razão de sua capacidade de evitar contaminação microbiológica significativa das amostras, o que não ocorreu com o biofilme de bagaço de uva. O uso de biofilmes em amostras sob refrigeração, por sua vez, se mostrou amplamente inadequado, pois prejudicou a eficácia da atuação do frio no processo de retardamento da perda de massa.

Conclui-se que a aplicação de biofilme de fécula de mandioca em tomates pode consistir em solução para prolongar sua vida útil em condições não refrigeradas e para evitar contaminação microbiológica. O biofilme de bagaço de uva, em razão de limitada ação de proteção microbiológica, se mostrou inferior e menos promissor em seu uso como cobertura.

\section{Referências}

BAUMGÄRTEL, T. et al. A note on digestibility and energy value for sheep of different grape pomace. Small Ruminant Research, v. 67, p. 302-306, 2007. https://doi.org/10.1016/j.smallrumres.2005.11.002.

CEREDA, M. P. Fécula de mandioca como ingrediente para alimentos. Revista da ABAM, Paranavaí, n. 11, 2005, s/p.

CHITARRA, M. I. F.; CHITARRA, A. B. Pós-colheita de frutos e hortaliças: fisiologia e manuseio. 2. Ed. Lavras: ESAL/FAEFE, 2005. 785p.

DEAMICI, K.M. et al. Drying kinetics of fermented grape pomace: determination of moisture effective diffusivity. Revista Brasileira de Engenharia Agrícola e Ambiental (Online), v. 20, p. 763-768, 2016. https://doi.org/10.1590/18071929/agriambi.v20n8p763-768.

DENG, Q.; PENNER, M.H.; ZHAO, Y. Chemical composition of dietary fiber and polyphenols of wine grape pomace skins and development of wine grape (cv. Merlot) pomace extract based films. Food Research International, v.44, p. 2712-2720, 2011. https://doi.org/10.1016/j.foodres.2011.05.026.

DOWNES, F. P.; ITO, H. Compendium of methods for the microbiological examination of foods. 4. ed. Washington: American Public Health Association (APHA), 2001. 676p. 
FAKHOURI, F. M. et al. Filmes e coberturas comestíveis compostas à base de amidos nativos e gelatina na conservação e aceitação sensorial de uvas Crimson. Ciência e Tecnologia de Alimentos, v. 27, p. 369-374, 2007. https://doi.org/10.1590/S010120612007000200027.

JIANG, Y.; SIMONSEN.; ZHAO, Y. Compression-molded biocomposite boards from red and white wine grape pomaces. Journal of Applied Polymer Science, v.119, n. 5, p. 2834-2846, 2011.

LEMOS, O.L. et al. Conservação do pimentão 'magali R' em duas condições de armazenamento associada à atmosfera modificada. Magistra, v. 20, n. 1, p. 06-15, 2008.

LUVIELMO, M.M.; LAMAS S.V. Revestimentos comestíveis em frutas. Estudos Tecnológicos em Engenharia, v. 8, n. 1, p.8-15, 2013. https://doi.org/10.4013/ete.2012.81.02.

MARCOS, S.R. Desenvolvimento de tomate de mesa, com o uso do método Q. F. P. (Quality Function Deployment) comercializado em um supermercado. 2001. 200 f. Tese (Doutorado em Tecnologia Pós-Colheita), Faculdade de Engenharia Agrícola, Universidade Estadual de Campinas, Campinas, 2001.

OLIVEIRA, D. A. de. Caracterização fitoquímica e biológica de extratos obtidos de bagaço de uva (Vitis vinifera) das variedades Merlot e Syrah. 2010. $211 \mathrm{f}$. Dissertação (Mestrado em Engenharia de Alimentos, Centro Tecnológico, Universidade Federal de Santa Catarina, Florianópolis, 2010.

OLIVEIRA, E.N.A. et al. Armazenamento de tomates revestidos com pectina: avaliação colorimétrica. Revista Caatinga. v. 25, n .4, p. 19-25, 2012. Disponível em: $<$ https://periodicos.ufersa.edu.br/index.php/caatinga/article/view/2606>. Acesso 28 dez. 2020.

PETRIKOSKI. A.P. Elaboração de biofilmes de fécula de mandioca e avaliação do seu uso na imobilização de caulinita intercalada com ureia. 2013. $129 \mathrm{f}$. Dissertação (Mestrado em Tecnologia de Processos Químicos e Bioquímicos), PósGraduação em Tecnologia de Processos Químicos e Bioquímicos, Universidade Tecnológica Federal do Paraná, Pato Branco, 2013.

REIS, L.C.B. et al. Filme biodegradável incorporado com glicerol e aditivos naturais. Cadernos de Prospecção, v. 4, n. 4, p.23-32, 2011. https://doi.org/10.9771/S.CPROSP.2011.004.003.

ROJAS-GRAÜ, M.A.; SOLIVA-FORTUNY, R.; MARTÍN-BELLOSO, O. Edible coatings to incorporate active ingredients to fresh-cut fruits: a review. Journal Trends of Food

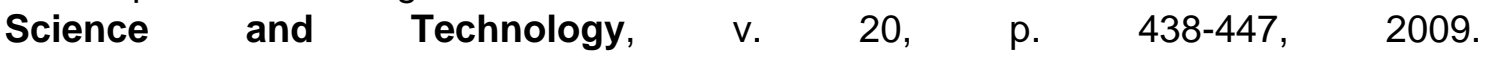
https://doi.org/10.1016/j.tifs.2009.05.002.

RUGGIERI, L. et al. Recovery of organic wastes in the Spanish wine industry. Technical, economic and environmental analyses of the composting process. Journal $\begin{array}{lllll}\text { of Cleaner Production, } & \text { v.17, } & \text { p. 830-838, }\end{array}$ https://doi.org/10.1016/j.jclepro.2008.12.005.

SILVA, D.F.P. et al. Desempenho de filmes comestíveis em comparação ao filme de policloreto de vinila na qualidade pós-colheita de mexericas 'Poncã'. Ciência Rural, v.42, n.10, p.1770-1773, 2012. https://doi.org/10.1590/S0103-84782012005000076. 
SOBRAL, P.J.A. Propriedades funcionais de biofilmes de gelatina em função da espessura. Ciência \& Engenharia, v. 8, n. 1, p. 60-67, 1999.

\section{Autores}

Elizangela Gonçalves de Oliveira*, Tatiana Valesca Rodriguez Alicieo

Centro de Ciências Químicas, Farmacêuticas e de Alimentos, Universidade Federal de Pelotas, Campus Universitário, Caixa Postal, 354, 96010-900, Pelotas, RS, Brasil.

*Autor para a correspondência: elizangelagoliveira1@gmail.com 